\title{
Comparative transcriptomics provide insight into the morphogenesis and evolution of fistular leaves in Allium
}

\author{
Siyuan Zhu, Shouwei Tang, Zhijian Tan, Yongting Yu, Qiuzhong Dai and Touming Liu*
}

\begin{abstract}
Background: Fistular leaves frequently appear in Allium species, and previous developmental studies have proposed that the process of fistular leaf formation involves programmed cell death. However, molecular evidence for the role of programmed cell death in the formation of fistular leaf cavities has yet to be reported.

Results: In this study, we characterized the leaf transcriptomes of nine Allium species, including six fistular- and three solid-leaved species. In addition, we identified orthologous genes and estimated their Ka and Ks values, in order to ascertain their selective pattern. Phylogenetic analysis based on the transcriptomes revealed that $A$. tuberosum was the most ancestral among the nine species, and analysis of orthologous genes between A. tuberosum and the other eight species indicated that 149 genes were subject to positive selection; whereas >3000 had undergone purifying selection in each species.

Conclusions: We found that many genes that are potentially related to programmed cell death either exhibited rapid diversification in fistular-leaved species, or were conserved in solid-leaved species in evolutionary history. These genes potentially involved in programmed cell death might play important roles in the formation of fistular leaf cavities in Allium, and the differing selection patterns in fistular- and solid-leaved species may be responsible for the evolution of fistular leaves.
\end{abstract}

Keywords: Allium, Transcriptome, Fistular leaf, Selective pattern, Programmed cell death

\section{Background}

As one of the largest genera of the petaloid monocotyledons, Allium (Amaryllidaceae) comprises more than 920 species [1] and includes several economically important crops that are cultivated for consumption or medicinal uses, such as garlic (A. sativum), welsh onion (A. fistulosum), leek (A. porrum), Chinese chives (A. tuberosum), onion (A. сера), Chinese jiaotou ( $A$. chinense), and shallot (A. ascalonicum). Varied leaf shapes can be observed among Allium species, including flat, columnar, solid, and fistular morphologies. Morphological and cellular studies have found that fistular leaves develop from solid precursors [2]. Developmental investigation of the leaves of A. fistulosum found that the process of fistular leaf formation involved programmed cell death (PCD)

\footnotetext{
* Correspondence: liutouming@caas.cn

Institute of Bast Fiber Crops and Center of Southern Economic Crops,

Chinese Academy of Agricultural Sciences, Changsha, China
}

[2]. PCD is a spontaneous, programmed, self-destructive cellular process that plays a key role in tissue differentiation, homeostasis, and organ morphogenesis, including that of leaves [3-5]. However, molecular evidence for the involvement of PCD in the formation of fistular leaf cavities is still absent in Allium, owing to the limited availability of genetic resources for Allium spp.

The paucity of genetic resources in Allium is, in part, due to the fact that Allium spp. have the largest genomes among eukaryotes $[6,7]$, with genome sizes ranging from 6860 to $30,870 \mathrm{Mbp}$ per C [8]. The enormous size of these genomes has been a major obstacle for their characterization and for gene mining in the family as a whole. In the past 10 years, the next generation sequencing technologies have undergone rapid development, and more than 80 plant species have had their complete genome [9]. However, none of genome of Allium species has been characterized, because of their enormous size. 
Because transcriptome analysis by next generation sequencing is rapid, inexpensive, and unconstrained by genomic complexity, it has been widely used as a primary tool for gene discovery and expression profiling in hundreds of plant species $[10,11]$. Moreover, transcriptome analysis can also be used as an important tool for investigating the domesticated patterns of crops $[12,13]$, as well as for investigating the mechanisms of development for specific traits [14]. In Allium, the transcriptome of four species, i.e. garlic [15, 16], onion [17], Chinese chive [18], and welsh onion [19], have been sequenced and de novo assembled. Among these, onion and Welsh onion are fistular-leaved species, whereas the other two produce solid leaves. However, despite the large numbers of generated expressed sequence tags, the molecular mechanism for the formation of fistular leave cavities is still uncharacterized.

Therefore, we analyzed the transcriptomes of nine economically important Allium species, including eight vegetable species and one herbal species (A. macrostemon). Thereafter, genes that had undergone significant selection were identified in both fistular- and solid-leaf species, respectively, and the genes with evolutionary divergence between fistular- and solid-leaf species were screened. As a result, the current study presents molecular evidence regarding a potential evolutionary mechanism for the development of fistular leaves.

\section{Methods}

\section{Plant material and RNA extraction}

The current study utilized local varieties of nine economically important Allium species. Among the eight vegetable species, three $[A$. sativum (garlic; SAT), $A$. porrum (leek; POR), and $A$. tuberosum (Chinese chives; TUB)] possess flat, solid leaves, whereas four species, $A$. fistulosum (welsh onion; FIS), A. ascalonicum (shallot; ASC), A. cepa (onion; CEP), and $A$. cepa var. agrogarum (AGR), have cylindrical, fistular leaves, and $A$. chinense (Chinese jiaotou; $\mathrm{CHI}$ ) has triangular, fistular leaves (Fig. 1). In addition, the leaves of the herbal species $A$. macrostemon (MAC) are also fistular, but internal cavities are very small (Fig. 1). The varieties of SAT, CEP, and MAC were collected from Chaling (Hunan, China), whereas those of ASC, POR, TUB, and $\mathrm{CHI}$ were collected from Ningxiang (Changsha, China), and the varieties of FIS and AGR were collected from Yuanjiang (Hunan, China) and Fuyu (Jinlin, China), respectively. The transverse section of leaves of nine Allium species was observed by Nikon AZ100 microscope (Nikon, Toyota, Japan).

All the varieties were established in the experimental field of the Institute of Bast Fiber Crops, Chinese Academy of Agricultural Sciences, Changsha, China on Sept. 15, 2014, and on Mar. 10, 2015, leaf tissue was sampled from three individuals of each species, immediately frozen in liquid nitrogen, and stored at $-80^{\circ} \mathrm{C}$ until used. The total RNA of each sample was extracted using an EZNA. Plant RNA Kit (OMEGA Bio-Tek, Norcross, GA, USA), according to the manufacturer's protocol.

\section{cDNA library construction, sequencing, and assembly} Illumina sequencing for the nine species was performed at Novogene Bioinformatics Technology Co., Ltd (Beijing, China; www.novogene.com). First, the total

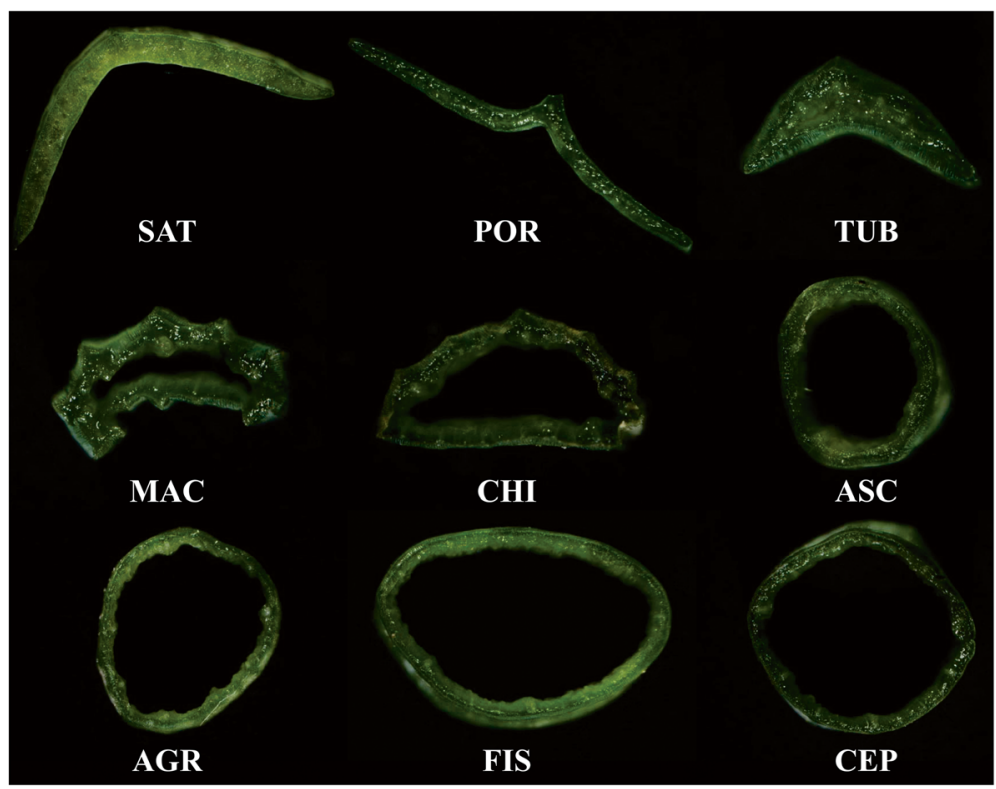

Fig. 1 The transverse section picture of leaves of nine Allium species 
RNA of each sample was used to construct a cDNA library with fragment lengths of $\sim 250 \mathrm{bp}$. Thereafter, paired-end sequencing was performed using the Illumina HiSeq 2500 sequencing platform (Illumina, San Diego, CA, USA), and the clean sequencing reads of each species were deposited in the NCBI Sequence Read Archive (Additional file 1: Table S1). After trimming the adapter sequences and filtering low-quality reads, the reads of each species were used to assemble their transcriptomes de novo using Trinity [20]. Sequences not extended on either end were categorized as unigenes. The sequences of unigenes assembled are deposited in the NCBI Transcriptome Shotgun Assembly (TSA) Database (Additional file 1: Table S1).

\section{Gene annotation}

To annotate the unigenes of each species, the corresponding sequences were searched against public databases, including the NCBI non-redundant protein sequences (NR) database, NCBI nucleotide sequences (NT) database, eukaryotic ortholog groups (KOG) database, Kyoto Encyclopedia of Genes and Genomes ortholog (KO) database, Swiss-Prot protein database, Gene Ontology (GO) database, and protein family (PFAM) database.

\section{Ortholog search and construction of phylogenetic tree}

The OrthoMCL method, which is based on the Markov cluster algorithm [21], was used to identify for orthologous genes among the nine species. According to the orthologous gene sequences, the phylogenetic relationships of nine species were reconstructed using Phyml 3.0, which is based on a Bayes algorithm [22], and the tree was visualized using MEGA 4.0 [23].

\section{$\mathrm{Ka} / \mathrm{Ks}$ analysis}

Positive selection is a process that favors the retention of beneficial mutations, as well as the rapid diversification of the affected genes. If a gene has evolved under positive selection, the sequence is expected to contain more non-synonymous nucleotide substitutions (Ka) than synonymous nucleotide substitutions (Ks) and, accordingly, should also exhibit a high $\mathrm{Ka} / \mathrm{Ks}$ ratio. In contrast, purifying selection is a process that removes deleterious alleles, and the sequences of genes subjected to purifying selection are relatively conserved. Accordingly, these sequences contain fewer, if any, non-synonymous substitutions, which results in a $\mathrm{Ka}$ that is much smaller than $\mathrm{Ks}$, and a low $\mathrm{Ka} / \mathrm{Ks}$ ratio. To identify genes subjected to significant selection, the Ka and Ks values were estimated using the Codeml program of Phylogenetic Analysis by Maximum Likelihood (PAML) based on the basic model [24], and genes with $\mathrm{Ka} / \mathrm{Ks}$ ratios $>1$ were considered as under positive selection, whereas genes with $\mathrm{Ka} / \mathrm{Ks}$ ratios $<0.1$ were considered as under purifying selection [25].

\section{Enrichment analysis}

The enrichment of GO functional categories in the selected genes of each species were analyzed using GOseq, which is based on the Wallenius non-central hypergeometric distribution [26], and enrichment analysis of the Kyoto Encyclopedia of Genes and Genomes (KEGG) pathways was performed using KOBAS [27]. Q values were used for determining the P-value threshold in multiple test and analysis [28], and pathways or GO categories with $\mathrm{Q}<0.05$ were considered significantly enriched.

\section{Results}

Transcriptome sequencing, assembly, and annotation Illumina paired-end sequencing of leaf transcriptomes from nine Allium spp. yielded about 99.9-128.9 million clean sequencing reads with a mean length of $\sim 125 \mathrm{bp}$ for each species (Table 1). Except for AGR, in which only 83,186 unigenes were de novo assembled, all species yielded $>117$ thousand unigenes, with an average length of 529-641 bp. POR had the largest transcriptome, at 109.28 Mb, whereas the transcriptomes of AGR and $\mathrm{CHI}$ were relatively small, at 53.35 and $66.84 \mathrm{Mb}$, respectively, and the other six species possessed

Table 1 Summary of transcriptome sequencing for nine Allium species

\begin{tabular}{|c|c|c|c|c|c|c|c|c|}
\hline Species & Abbr. & Crop names & Leaf shape & $\begin{array}{l}\text { Clean read number } \\
\text { (Million) }\end{array}$ & $\begin{array}{l}\text { Transcriptome } \\
\text { size }(\mathrm{Mb})\end{array}$ & $\begin{array}{l}\text { Unigene } \\
\text { number }\end{array}$ & $\begin{array}{l}\text { Mean length of } \\
\text { unigene }(b p)\end{array}$ & $\begin{array}{l}\text { Gene number } \\
\text { annotated }\end{array}$ \\
\hline A. sativum & SAT & Garlic & Flat solid leaf & 103.6 & 77.95 & 132,225 & 590 & $46341(35.04 \%)$ \\
\hline A. porrum & POR & Leek & Flat solid leaf & 128.9 & 109.28 & 189,713 & 576 & $48621(25.62 \%)$ \\
\hline A. tuberosum & TUB & Chinese chives & Flat solid leaf & 109.4 & 83.78 & 148,715 & 563 & 44532 (29.94\%) \\
\hline A. macrostemon & MAC & & Fistular leaf with small gap & 99.9 & 85.48 & 161,681 & 529 & $52129(32.24 \%)$ \\
\hline A. chinense & $\mathrm{CHI}$ & Chinese jiaotou & Triangular fistular leaf & 111.9 & 66.84 & 121,008 & 552 & 41908 (34.63\%) \\
\hline A. ascalonicum & ASC & Shallot & Columnar fistular leaf & 100.8 & 71.62 & 117,659 & 609 & 40315 (34.26\%) \\
\hline A. fistulosum & FIS & Welsh onion & Columnar fistular leaf & 121.0 & 76.56 & 128,372 & 596 & $42311(32.95 \%)$ \\
\hline A. сера L. var. сера & CEP & Onion & Columnar fistular leaf & 102.9 & 74.03 & 117,189 & 632 & $39472(33.68 \%)$ \\
\hline A. cepa var. agrogarum & $A G R$ & & Columnar fistular leaf & 102.5 & 53.35 & 83,186 & 641 & 34141 (41.04\%) \\
\hline
\end{tabular}


intermediate sized transcriptomes, ranging from 71.62 to $85.48 \mathrm{Mb}$ (Table 1). In addition, except for the unigenes of POR and AGR, of which 25.62 and $41.04 \%$ were successfully annotated, respectively, $\sim 30-35 \%$ of unigenes were typically annotated.

\section{Phylogenetic analysis}

According to our phylogenetic tree (Fig. 2), TUB was located at the root of the tree, and had far phylogenetic distance with the others, suggesting that TUB was more ancestral than the other species. This finding was consistent with the result of previous study [29]. The other eight species were split into four clades. Interestingly, the classification based on the transcriptomes was consistent with that based on leaf morphology, i.e., the species with flat, solid leaves (SAT and POR) were grouped together, the species with cylindrical, fistular leaves (ASC, FIS, CEP, and AGR) were placed in a monophyletic clade, and the species with small-gapped and triangular fistular leaves (MAC and $\mathrm{CHI}$ ) were placed in a single branch, as well (Fig. 2).

\section{Identification of genes subjected to significant selection}

A total of 29,990 orthologous groups were identified, with 9634 from the two flat, solid-leaved species (SAT and POR; Fig. 3a), 6995 from the four cylindrical, fistular-leaved species (ASC, FIS, CEP, and AGR; Fig. 3b), 3720 among all nine species (Fig. 3c).

Because the TUB transcriptome was ancestral to the transcriptomes of the other eight species, it was designated as the ancestral species in the present study. The orthologous genes between TUB and the other eight species were searched (Fig. 3d), and their Ka and Ks values were estimated and used to determine the selective pattern of them (Fig. 4). A total of 149 genes were found to undergo positive selection with 13 to 27 identified in each species (Fig. 5), suggesting that these 149 genes exhibited a rapid diversification in evolutionary history. Among these 149 positively selected genes, 39 genes (from 18 orthologous groups) were mitochondrial (Additional file 2: Table S2), 29 genes (from 13 orthologous groups) were chloroplast (Additional file 3: Table S3), and 81 genes (from 70 orthologous groups) were nuclear (Additional file 4: Table S4). In addition, $>3000$ genes were found to undergo purifying selection in each species (Fig. 5), which suggested that the sequences of these genes were relatively conserved, in evolutionary history.

\section{Enrichment analysis for selected genes}

None of the GO categories were enriched by positively selected genes in any species, although 51-72 terms were significantly enriched by genes subjected to purifying selection in each species $(\mathrm{Q}<0.05$; Additional file 5: Table S5). Among the enriched terms, 23 were enriched by genes of purifying selection in all eight species, suggesting that the functional terms are essential for growth in the species. In addition, one KEGG pathway, oxidative phosphorylation, exhibited significant enrichment by positively selected genes in all species, except FIS $(\mathrm{Q}<0.05)$; however, no pathways were markedly enriched by genes of purifying selection $(\mathrm{Q}<0.05)$.

\section{Identification of selected genes potentially related to PCD} Among 149 identified positively selected genes, 25 from 11 orthologous groups were annotated as proteins involved in plant PCD (Table 2), including 15 genes related to the metabolism of cytochrome $\mathrm{c}$, which is an important factor for inducing PCD [30], and two genes encoding PCD-related kinase proteins. In addition, there were 4970 orthologous groups in which the orthologous gene of at least two species had undergone purifying selection. Of the 4,970 orthologous groups, there were 116 groups in which only the orthologous genes from the flat, solid-leaved species(SAT and POR) were subjected to purifying selection (Additional file 6: Table S6), suggesting that the genes are possibly related to leaf morphology. Among the 116 orthologous groups, five were PCD-related (Table 3), especially OG01095 that encodes

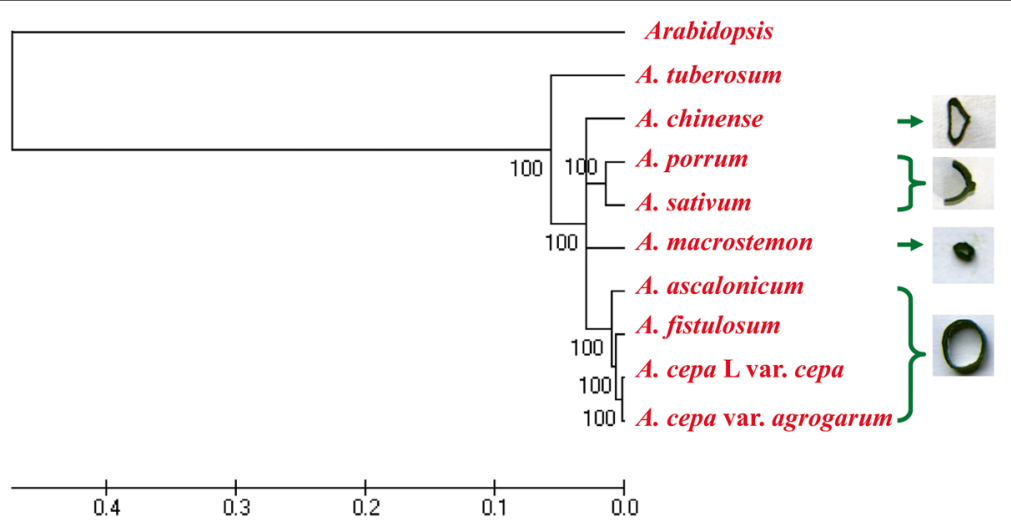

Fig. 2 Phylogenetic tree of nine Allium species, constructed on the basis of their transcriptomes 

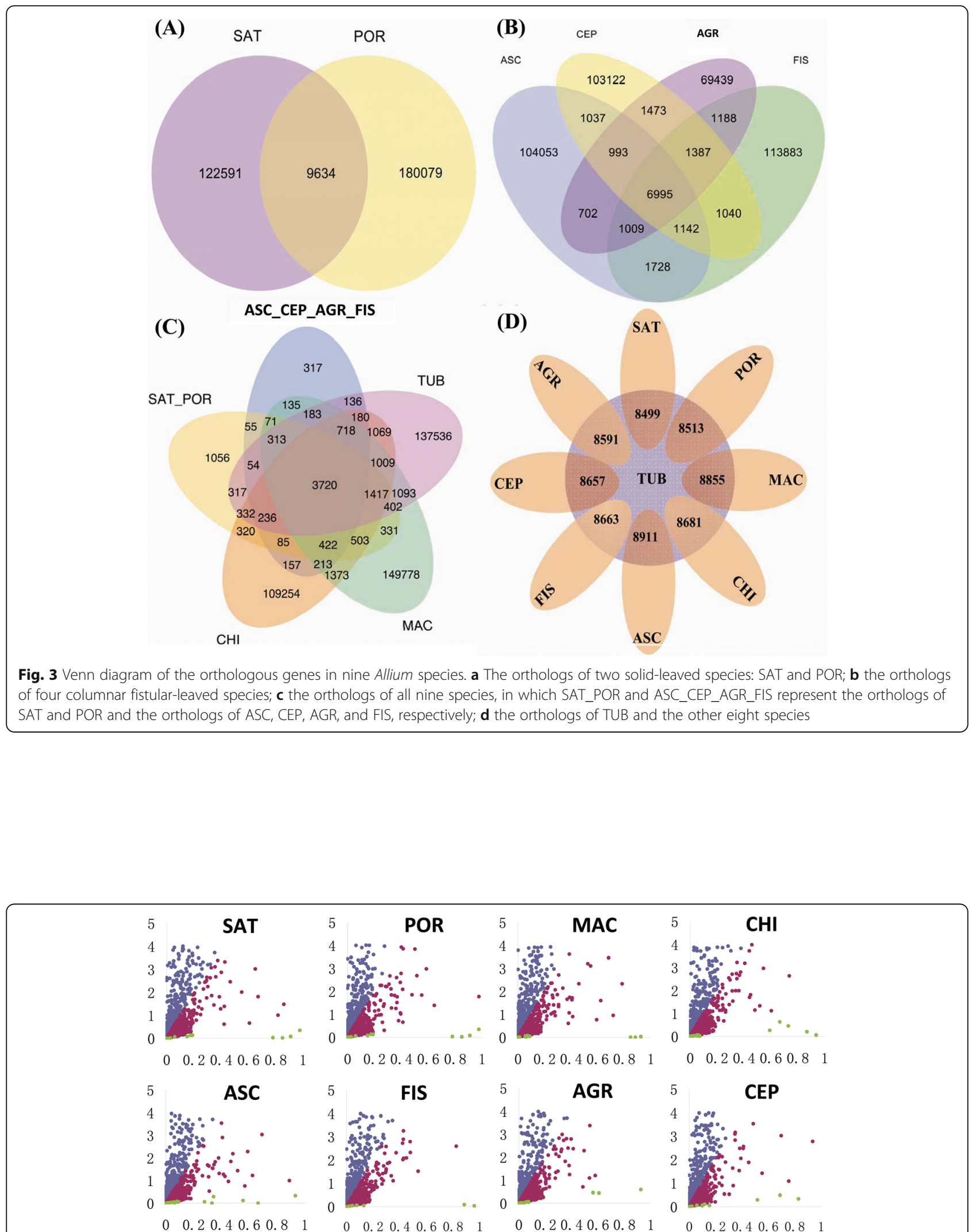

Fig. 4 Scatter diagrams of Ka and Ks values. The Ka and Ks values of the orthologs of TUB and the other eight species were estimated. The $x$ and y axes represent the Ka and Ks values, respectively. Orthologous genes with $\mathrm{Ka}>1$ or $\mathrm{Ks}>4$ are not shown 


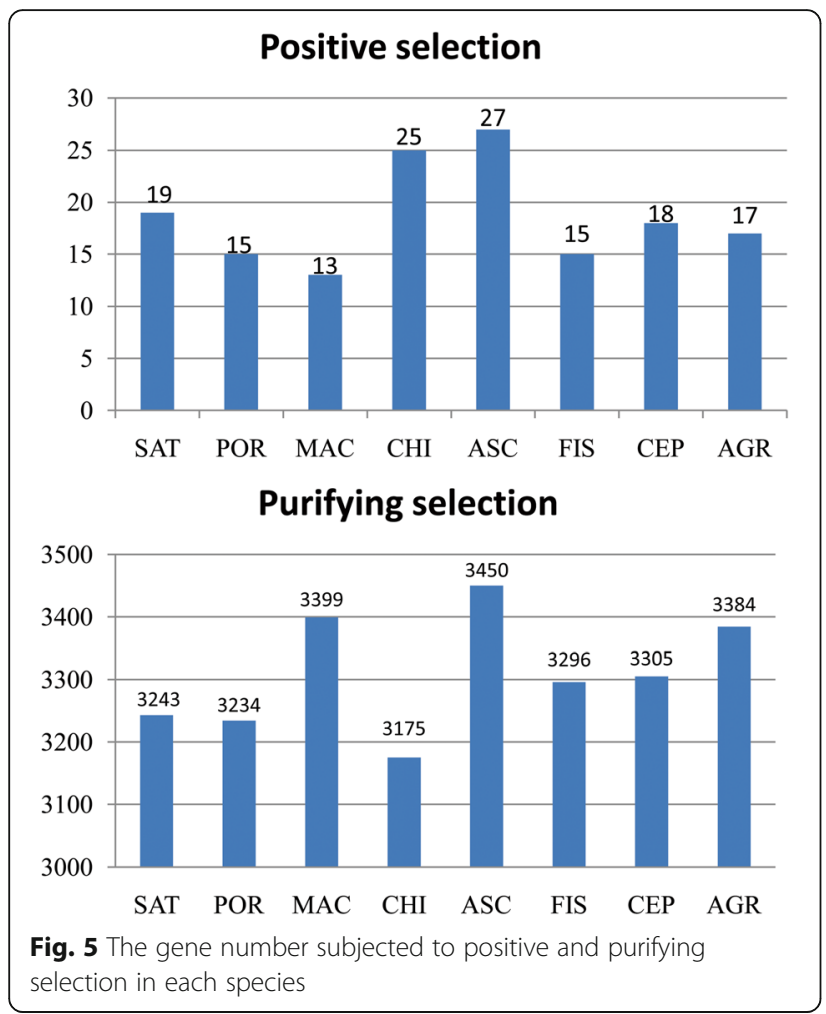

a metacaspase-like protein, which is a key regulator of plant PCD [31].

\section{Discussion}

Although Allium comprises a large number of species and even includes a few species of significant economic importance, the transcriptomes of only four species (i.e. garlic, onion, welsh onion, and Chinese chive) have been analyzed [15-19]. Therefore, in the present study, the transcriptomes of nine Allium species were sequenced and assembled de novo, and large numbers of genes were identified in each species. These results will help us investigate the major genes responsible for important agronomic traits in Allium species, and will also further our understanding of the mechanisms underlying the formation and development of such traits, including leaf morphology.

Leaves are the primary site of plant photosynthesis and respiration. Diverse leaf forms can be observed in angiosperms, and they can be categorized into two major categories: bifacial or unifacial. Development studies have proposed that the diversity of leaf morphology is caused by differential and asymmetrical growth along the adaxial-abaxial, central-marginal, and proximal-distal polarities [32-34]. Allium spp. exhibit a wide variety of leaf shapes, including cylindrical and hollow, flat and solid, and hollow and triangular types, the differences of which can be observed in transverse sections [35]. As in other angiosperms, there is a great possibility that the diversity of leaf appearance in Allium is caused by these three polarities. For example, only the abaxial identity existed in leaf blades results in a unifacial fistular leaf in Welsh onion [33, 34] However, the formation of fistular leaf cavities is still poorly understood. Although that a cellular investigation of the leaves of A. fistulosum found that the process of fistular leaf formation involved PCD [2], molecular evidences for the involvement of PCD in the formation of fistular leaf cavities is still unknown in Allium.

Previous studies have shown that mitochondria play a central role in controlling PCD in plants [30], by releasing molecules, including cytochrome $\mathrm{c}$ that drive the destruction of the cell [36]. In animals, cytochrome c triggers PCD by forming a complex that induces the formation of apoptosomes, and those apoptosomes then activate downstream effector caspases, which are key

Table 2 Positively selected genes potentially related to programmed cell death

\begin{tabular}{llll}
\hline Orthologous group & Positively selected genes & Gene source & Function annotated \\
\hline OG06438 & MAC|c99454_g1, CHI|c73660_g1, ASC|c61655_g1, & Mitochondrial genome & Cytochrome c oxidase subunit 3 \\
OG08411 & FIS|c70920_g1, CEP|c54051_g1, AGR|c38049_g1 & \\
OG12823 & MAC|c48312_g1, ASC|c92926_g1 & Mitochondrial genome & cytochrome c biogenesis Fn \\
OG13835 & ASC|c67719_g2, AGR|c41049_g1 & Mitochondrial genome & cytochrome c oxidase subunit 2 \\
OG20657 & SAT|c125661_g1, POR|c94489_g1, MAC|c79087_g1 & Mitochondrial genome & cytochrome c maturation protein CcmB \\
OG04672 & CEP|c5005_g1, AGR|c29721_g1 & Mitochondrial genome & cytochrome c biosynthesis ccmC-like protein \\
OG27563 & SAT|c121207_g1, MAC|c150852_g1, ASC|c70366_g1, & Chloroplastic Genome & photosystem II protein D1 \\
OG15984 & FIS|c65166_g1 & Chloroplastic Genome & photosystem II protein \\
OG22529 & ASC|c29090_g1 & Nuclear Genome & Serine/threonine-protein kinase ATM-like protein \\
OG28738 & FIS|c46048_g1, CEP|c60376_g1 & Nuclear Genome & serine/threonine-protein kinase ATR-like protein \\
OG09355 & CHI|c103126_g1 & Nuclear Genome & KDEL-tailed cysteine endopeptidase \\
\hline
\end{tabular}


Table 3 Programmed cell death-related orthologous groups in which only the orthologous genes from the flat, solid-leaved species A. sativum and A. porrum were subjected to purifying selection

\begin{tabular}{llll}
\hline Orthologous group & Genes of purifying selection & Gene source & Function annotated \\
\hline OG02476 & POR|c97456_g1, SAT|c68586_g1 & Nuclear Genome & mitogen-activated protein kinase 9-like \\
OG01095 & SAT|c13216_g1, POR|c105213_g1 & Nuclear Genome & metacaspase-4-like \\
OG03448 & SAT|c76384_g1, POR|c107735_g1 & Nuclear Genome & MACPF domain-containing protein CAD1 \\
OG10721 & SAT|C83301_g1, POR|c11731_g1 & Chloroplastic Genome & photosystem II protein, chloroplast \\
OG23573 & SAT|c138076_g1, POR|c7088_g1 & Chloroplastic Genome & photosystem II P680 reaction center D1 protein, chloroplast \\
\hline
\end{tabular}

regulators of PCD [30]. Although no caspase homologs have been found in plants, caspase-like proteins have been described in cells undergoing PCD, such as the cysteine endopeptidases, which can be subdivided into two groups: vacuolar processing enzymes and metacaspases $[37,38]$. In the present study, we identified 15 positively selected genes from five orthologous groups that were potentially related to the metabolism of cytochrome $\mathrm{c}$ and were found to undergo positive selection. Among these 15 genes, 12 were subjected positive selection in fistular-leaved species, except 3 genes from OG13835 that underwent positive selection in the species with solid or small-gapped fistular leaves. In addition, a KDEL-tailed cysteine endopeptidase-encoding gene from the fistularleaved ASC exhibited rapid evolution, and a metacaspase4-like protein-encoding gene was found to undergo positive selection in solid-leaved species, but evolved neutrally in fistular-leaved species.

Like the mitochondria, the chloroplasts are also important in triggering PCD, by mediating reactive oxygen species signaling. In plants, photosynthesis is performed in photosystem II of chloroplasts. When photosynthesis is less efficient, or chloroplasts receive excessive levels of light, chloroplasts produce more reactive oxygen species, and then the reactive oxygen species drives cell death finally $[30,39,40]$. In the present study, we identified five chloroplast genes that are potentially involved in photosynthesis in photosystem II and exhibited rapid diversification. Among them, four of the genes were from fistular-leaved species, and one was from a flat, solid-leaved species (i.e., SAT). In addition, two photosystem II protein-encoding genes were also found to have undergone purfying selection in solid-leaved species, whereas they were evolving neutrally in fistular-leaved species.

After the "death signal" is generated by mitochondria and/or chloroplasts, it is transduced by kinase proteins. Previous studies have shown that PCD signaling involves the activation of mitogen-activated protein kinases that induce hypersensitive responses and cell apoptosis [41]. Recently, the phosphatidyl-3 kinases ATM and ATR were reported as the main sensors of DNA damageinduced PCD $[42,43]$. In the present study, the ATM and ATR kinase-encoding genes in the fistular-leaved species FIS, CEP, and CHI exhibited evidence of rapid diversification, but not in solid-leaved species, and mitogen-activated protein kinase-encoding gene underwent positive selection in solid-leaved species, whereas it was evolving neutrally in fistular-leaved species.

In addition, two genes, LSD-one-like protein 2 (LOL2) and constitutively activated cell death 1 (CAD1), have also been reported to play important roles in regulating PCD in Arabidopsis [44, 45]. In the present study, the orthologs of these two genes were also found to exhibit different selective patterns among the fistular- and solidleaved species. These potential PCD-related genes with evolutionary divergence might have roles in regulating the formation of cavity of fistular leaf in Allium species.

\section{Conclusion}

In this study, we compared the leaf transcriptomes of six fistular- and three solid-leaf species of Allium. The selective patterns of orthologous genes in nine species were analyzed, and the result showed that many mitochondrial, chloroplast and nuclear genes that were potentially involved in plant PCD and were either subject to positive selection in fistular-leaved species but not in solid-leaved species, or evolved neutrally in fistularleaved species but purifying selection in solid-leaved species. These potential PCD-related genes might play roles in the formation of the inner cavities of fistular leaves in Allium, and the differing selection patterns in fistular- and solid-leaved species may be responsible for the evolution of fistular leaves.

\section{Additional files}

Additional file 1: Table $\mathbf{S 1}$. Total length of clean sequencing reads from each species and their accession numbers in database. (DOCX 16 kb)

Additional file 2: Table S2. Positively selected mitochondrial genes in evolution. (DOCX $13 \mathrm{~kb}$ )

Additional file 3: Table S3. Positively selected chloroplast genes in evolution. (DOCX 13 kb)

Additional file 4: Table S4. Positively selected nuclear genes in evolution. (XLSX $14 \mathrm{~kb}$ )

Additional file 5: Table S5. Table S5 Gene ontology (GO) terms enriched by genes of purifying selection in each species. (XLSX 16 kb)

Additional file 6: Table S6. Orthologous groups in which only the orthologous genes from the flat, solid-leaved species $A$. sativum and $A$. porrum were subjected to purifying selection. (XLSX $17 \mathrm{~kb}$ ) 


\section{Abbreviations}

AGR: A. cepa var. agrogarum; ASC: A. ascalonicum; CEP: A. cepa; CHI: A. chinense; FIS: A. fistulosum; GO: Gene Ontology database; Ka: Non-synonymous nucleotide substitutions; KO: KEGG ortholog database; KOG: Eukaryotic ortholog groups database; Ks: Synonymous nucleotide substitutions; MAC: A. macrostemon NR: NCBI non-redundant protein sequencesdatabase; NT: NCBI nucleotide sequences database; PCD: Programmed cell death; PFAM: Protein family database; POR: A. porrum; SAT: A. sativum; TUB: A. tuberosum

\section{Acknowledgements}

We acknowledge the Novogene Bioinformatics Institute for its assistance in original data processing and related bioinformatics analysis.

\section{Funding}

This work was supported by grants from the Agricultural Science and Technology Innovation Program of China (CAAS-ASTIP-IBFC). The funders had no role in study design, data collection and analysis, decision to publish, or preparation of the manuscript.

\section{Availability of data and materials}

The sequencing reads is available in the NCBI SRA database under accession SRX1560526, SRX1560538, SRX1560563, SRX1560658, SRX1560673, SRX1560692, SRX1560712, SRX1560726, and SRX1560727; the transcripts assembled are deposited in the NCBI Transcriptome Shotgun Assembly (TSA) Database under accession number GEOY00000000, GFAP00000000, GFAR00000000, GFAN00000000, GFAO00000000, GFAL00000000, GFAJ00000000, GFAK00000000, GFAM00000000

\section{Authors' contributions}

SZ and QD performed the experiment. SZ revised the manuscript, and submitted the data to database. ST planned and designed the research. YY conducted the fieldwork, and ZT prepared the reagents of experiments. $\mathrm{TL}$ analyzed the data and wrote the manuscript. All authors read and approved the final manuscript.

\section{Competing interests}

The authors declare that they have no competing interests.

\section{Consent for publication}

Not applicable.

\section{Ethics approval and consent to participate} Not applicable.

\section{Received: 13 May 2016 Accepted: 26 December 2016}

Published online: 10 January 2017

\section{References}

1. Herden T, Hanelt P. Friesen N. Phylogeny of Allium L. subgenus Anguinum (G. Don. ex W.D.J. Koch) N. Friesen (Amaryllidaceae). Mol Phylogenet Evol. 2016;95:79-93.

2. Ni X, Su H, Zhou Y, Wang F, Liu W. Leaf-shape remodeling: programmed cell death in fistular leaves of Allium fistulosum. Physiol Plant. 2015;153:419-31.

3. Meier P, Finch A, Evan G. Apoptosis in development. Nature. 2000:407:796-801.

4. Gunawardena A, Greenwood J, Dengler N. Programmed cell death remodels lace plant leaf shape during development. Plant Cell. 2004;16:60-73.

5. Gunawardena A, Sault K, Donnelly P, Greenwood J, Dengler N. Programmed cell death and leaf morphogenesis in Monstera obliqua (Araceae). Planta. 2005;221:607-18.

6. Labani R, Elkington T. Nuclear DNA variation in the genus Allium L. (Liliaceae). Heredity. 1987;59:119-28.

7. Baranyi M, Greilhuber J. Genome size in Allium: in quest of reproducible data. Ann Bot. 1999:83:687-95.

8. Ricroch A, Yockteng R, Brown SC, Nadot S. Evolution of genome size across some cultivated Allium species. Genome. 2005:48:511-20.

9. Kitts P, Church D, Thibaud-Nissen F, Choi J, Hem V, Sapojnikov V, Smith R, Tatusova T, Xiang C, Zherikov A, et al. Assembly: a resource for assembled genomes at NCBI. Nucleic Acids Res. 2016;44:D73-80.

10. Liu T, Zhu S, Tang Q, Chen P, Yu Y, Tang S. De novo assembly and characterization of transcriptome using Illumina paired-end sequencing and identification of CesA gene in ramie (Boehmeria nivea L. Gaud). BMC Genomics. 2013:14:125.

11. Mutz K, Heilkenbrinker A, Lonne M, Walter J, Stahl F. Transcriptome analysis using next-generation sequencing. Curr Opin Biotechnol. 2013;24:22-30.

12. Liu T, Tang S, Zhu S, Tang Q, Zheng X. Transcriptome comparison reveals the patterns of selection in domesticated and wild ramie (Boehmeria nivea L. Gaud). Plant Mol Biol. 2014;86:85-92.

13. Koenig D, Jiménez-Gómez J, Kimura S, Fulopa D, Chitwooda D, Headlanda L, Kumara R, Covingtona M, Devisettya U, Tata A, et al. Comparative transcriptomics reveals patterns of selection in domesticated and wild tomato. Proc Natl Acad Sci U S A. 2013;110:E2655-62.

14. Lou Q, Liu Y, Qi Y, Jiao S, Tian F, Jiang L, Wang Y. Transcriptome sequencing and metabolite analysis reveals the role of delphinidin metabolism in flower colour in grape hyacinth. J Exp Bot. 2014;65:3157-64

15. Kamenetsky R, Faigenboim A, Mayer E, Michael T, Gershberg C, Kimhi S, Esquira I, Shalom S, Eshel D, Rabinowitch $H$, et al. Integrated transcriptome catalogue and organspecific profiling of gene expression in fertile garlic (Allium sativum L.). BMC Genomics. 2015;16:12

16. Liu T, Zeng L, Zhu S, Chen X, Tang Q, Mei S, Tang S. Large-scale development of expressed sequence tag-derived simple sequence repeat markers by deep transcriptome sequencing in garlic (Allium sativum L.). Mol Breed. 2015;35:204.

17. Rajkumar H, Ramagoni R, Anchoju V, Vankudavath R, Syed A. De novo transcriptome analysis of Allium cepa $\mathrm{L}$. (Onion) bulb to identify allergens and epitopes. PLoS One. 2015;10:e0135387.

18. Zhou S, Chen L, Liu S, Wang X, Sun X. De novo assembly and annotation of the Chinese chive (Allium tuberosum Rottler ex Spr.) transcriptome using the Illumina platform. PLoS One. 2015:10:e0133312.

19. Sun $X, Y u X$, Zhou S, Liu S. De novo assembly and characterization of the Welsh onion (Allium fistulosum L.) transcriptome using Illumina technology. Mol Genet Genomics. 2016;291:647-59.

20. Grabherr M, Haas B, Yassour M, Levin J, Thompson D, Amit I, Adiconis X, Fan $L$, Raychowdhury R, Zeng $Q$, et al. Full length transcriptome assembly from RNA-Seq data without a reference genome. Nat Biotechnol. 2011;29:644-52.

21. Li L, Stoeckert Jr C, Roos D. OrthoMCL: Identification of ortholog groups for eukaryotic genomes. Genome Res. 2003;13:2178-89.

22. Guindon S, Dufayard J, Lefort V, Anisimova M, Hordijk W, Gascuel O. New algorithms and methods to estimate maximum-likelihood phylogenies: assessing the performance of PhyML 3.0. Syst Biol. 2010;59:307-21.

23. Tamura K, Dudley J, Nei M, Kumar S. MEGA4: molecular evolutionary genetics analysis (MEGA) software version 4.0. Mol Biol Evol. 2007;24:1596-9.

24. Yang Z. PAML 4: Phylogenetic analysis by maximum likelihood. Mol Biol Evol. 2007:24:1586-91.

25. Shah C, VanGompel MJW, Naeem V, Chen Y, Lee T, Angeloni N, Wang Y, XU E. Widespread presence of human BOULE homologs among animals and conservation of their ancient reproductive function. PLoS Genet. 2010;6: e1001022

26. Young M, Wakefield M, Smyth G, Oshlack A. Gene ontology analysis for RNA-seq: accounting for selection bias. Genome Biol. 2010;11:R14.

27. Xie C, Mao X, Huang J, Ding Y, Wu J, Dong S, Kong L, Gao G, Li C, Wei L. KOBAS 2.0: a web server for annotation and identification of enriched pathways and diseases. Nucleic Acids Res. 2011;39:W316-22.

28. Benjamini $Y$, Hochberg $Y$. Controlling the false discovery rate: a practical and powerful approach to multiple testing. J Roy Stat Soc B. 1995;57:289-300.

29. Friesen N, Fritsch $R$, Blattner F. Phylogeny and new intrageneric classification of Allium (Alliaceae) based on nuclear ribosomal DNA ITS sequences. Aliso. 2006;22:372-95.

30. Reape T, Brogan N, McCabe P. Mitochondrion and chloroplast regulation of plant programmed cell death. In: Gunawardena A, McCabe P, editors. Plant Programmed Cell Death. New York: Springer; 2015. p. 33-53.

31. Lam E, Zhang Y. Regulating the reapers: activating metacaspases for programmed cell death. Trends Plant Sci. 2012:17:487-94.

32. Yamaguchi $\mathrm{T}$, Tsukaya $\mathrm{H}$. Evolutionary and developmenta studies of unifacial leaves in monocots: Juncus as a model system. J Plant Res. 2010;123:35-41

33. Yamaguchi T, Nukazuka A, Tsukaya H. Leaf adaxial-abaxial polarity specification and lamina outgrowth: evolution and development. Plant Cell Physiol. 2012;53:1180-94.

34. Yamaguchi T, Yano S, Tsukaya H. Genetic framework for flattened leaf blade formation in unifacial leaves of Juncus prismatocarpus. Plant Cell. 2010:22: 2141-55. 
35. Lee SH. A comparative anatomical study on the leaves of some bulb crops in China. J Integr Plant Biol. 1952;1:1-7.

36. Diamond M, McCabe P. Mitochondrial regulation of plant programmed cell death. In: Kempken F, editor. Plant mitochondria, vol 1, Advances in plant biology. New York: Springer; 2011. p. 439-65.

37. Helm M, Schmid M, Hierl G, Terneus K, Tan L, Lottspeich F, Kieliszewski M, Gietl C. KDEL-tailed cysteine endopeptidases involved in programmed cell death, intercalation of new cells, and dismantling of extension scaffolds. Am J Bot. 2008;95:1049-62.

38. Lord C, Dauphinee A, Watts R, Gunawardena. A unveiling interactions among mitochondria, caspase-Like proteases, and the actin cytoskeleton during plant programmed cell death (PCD). PLoS ONE. 2013;8:e57110.

39. Kim C, Meskauskiene R, Zhang S, Lee K, Ashok M, Blajecka K, Herrfurth C, Feussner I, Apela K. Chloroplasts of Arabidopsis are the source and a primary target of a plant-specific programmed cell death signaling pathway. Plant Cell. 2012;24:3026-39.

40. Aken O, Breusegem F. Licensed to kill: mitochondria, chloroplasts, and cell death. Trends Plant Sci. 2015;20:754-66.

41. Kuriyama H, Fukuda H. Developmental programmed cell death in plants. Curr Opin Plant Biol. 2002;5:568-73.

42. Amiard S, Depeiges A, Allain E, White C, Gallego M. Arabidopsis ATM and ATR kinases prevent propagation of genome damage caused by telomere dysfunction. Plant Cell. 2011;23:254-4265.

43. Smetana O, Siroky J, Houlne G, Opatrny Z, Chaboute M. Non-apoptotic programmed cell death with paraptotic-like features in bleomycin-treated plant cells is suppressed by inhibition of ATM/ATR pathways or NtE2F overexpression. J Exp Bot. 2012;63:2631-44.

44. Morita-Yamamuro C, Tsutsui T, Sato M, Yoshioka H, Tamaoki M, Ogawa D, Matsuura H, Yoshihara T, Ikeda A, Uyeda I, et al. The Arabidopsis gene CAD1 controls programmed cell death in the plant immune system and encodes a protein containing a MACPF domain. Plant Cell Physiol. 2005;46:902-12.

45. Coll N, Epple P, Dangl J. Programmed cell death in the plant immune system. Cell Death Differ. 2011;18:1247-56.

\section{Submit your next manuscript to BioMed Central and we will help you at every step:}

- We accept pre-submission inquiries

- Our selector tool helps you to find the most relevant journal

- We provide round the clock customer support

- Convenient online submission

- Thorough peer review

- Inclusion in PubMed and all major indexing services

- Maximum visibility for your research

Submit your manuscript at www.biomedcentral.com/submit

) Biomed Central 